Cavendish-HEP-2003/08

July 2003

\title{
The Influence of the Experimental Methodology on the QED Theoretical Uncertainties on the Measurement of $M_{\mathrm{W}}$ at LEP
}

\author{
M. A. Thomson \\ Cavendish Laboratory, University of Cambridge, \\ Cambridge CB3 OHE, England.
}

\begin{abstract}
Previous studies of the QED systematic uncertainties on the LEP measurement of the W-boson mass, $M_{\mathrm{W}}$, have used idealized event selections and fitting procedures. In this paper, the Monte Carlo tandem of KoralW and YFSWW is used to investigate how the full experimental $M_{\mathrm{W}}$ extraction procedure affects these estimates. It is found that the kinematic fitting used in the experimental determination of $M_{\mathrm{W}}$ enhances the sensitivity to QED corrections involving real photon production. It is concluded that the previous estimates of the QED theoretical uncertainty on the LEP2 $M_{\mathrm{W}}$ measurement may be too small. A simple procedure for approximating the effect of the kinematic fit at the level of the generated four fermions is proposed. This procedure would allow previous theoretical studies to be repeated using a much closer approximation of the real experimental mass extraction method. Finally, the possibility of setting experimental limits on $\mathcal{O}(\alpha)$ theoretical uncertainties using identified $\mathrm{e}^{+} \mathrm{e}^{-} \rightarrow \mathrm{W}^{+} \mathrm{W}^{-} \gamma$ events is discussed.
\end{abstract}




\section{Introduction}

It is anticipated that the final experimental error on the mass of the $\mathrm{W}$ boson, $M_{\mathrm{W}}$, from LEP 2 will be approximately $35 \mathrm{MeV}$. A potential source of theoretical uncertainty is the treatment of QED and electroweak corrections in the Monte Carlo programs used to simulate the process $\mathrm{e}^{+} \mathrm{e}^{-} \rightarrow 4 f(\gamma)$. In recent years $\mathcal{O}(\alpha)$ electroweak corrections have been implemented in the YFSWW[1] and RacoonWW[2] programs. The current estimate of the theoretical systematic uncertainty on the LEP measurement of $M_{\mathrm{W}}$ due to these predominantly QED corrections is less than $5 \mathrm{MeV}[3]$. However, as pointed out by the authors, this estimate is based on an idealized $M_{\mathrm{W}}$ extraction procedure. Indeed, the authors suggest that the study should be repeated using the full experimental fitting procedure.

In this paper the concurrent Monte Carlo combination[4] of KoralW[5] and YFSWW[1] (referred to as KandY) is used to study the sensitivity of the full experimental analysis to $\mathcal{O}(\alpha)$ corrections. Monte Carlo events generated using KandY are passed through the OPAL detector simulation[6] and W-boson reconstruction algorithm[7]. In this way, the previous estimates of the QED systematic uncertainties can be extended to the full experimental $M_{\mathrm{W}}$ extraction procedure. It is found that the sensitivity to the so-called[3] $\mathcal{O}(\alpha)$ non-leading (NL) electroweak corrections is significantly enhanced by the kinematic fitting used in the experimental analyses. This enhancement is a result of the relatively large change in the cross section for production of $\mathrm{e}^{+} \mathrm{e}^{-} \rightarrow \mathrm{W}^{+} \mathrm{W}^{-} \gamma$ events where the photon is away from the region collinear with the $\mathrm{e}^{+} \mathrm{e}^{-}$ beams. Consequently, measurements of the cross section for $\mathrm{e}^{+} \mathrm{e}^{-} \rightarrow \mathrm{W}^{+} \mathrm{W}^{-} \gamma$ in this region place constraints on the related $M_{\mathrm{W}}$ systematic uncertainties.

It is suggested that previous studies[3] be repeated using a closer approximation of the real experimental procedure. With this in mind, a simple procedure to approximate the effects of the kinematic fit at the level of the generated four fermions is proposed.

\section{The Experimental Measurement of $M_{\mathrm{W}}$ at LEP 2}

The main measurements of $M_{\mathrm{W}}$ at LEP are obtained from the direct reconstruction of the W-boson invariant mass distribution in $\mathrm{e}^{+} \mathrm{e}^{-} \rightarrow \mathrm{q} \overline{\mathrm{q}} \ell \bar{\nu}_{\ell}$ and $\mathrm{e}^{+} \mathrm{e}^{-} \rightarrow \mathrm{q} \overline{\mathrm{q}} \overline{\mathrm{q}}$ events. Although the exact methodologies used by the four LEP experiments differ[7, 8], there are many common features and the general principles of the analyses are summarized below.

The experimental event selections for $\mathrm{e}^{+} \mathrm{e}^{-} \rightarrow \mathrm{W}^{+} \mathrm{W}^{-}$have high efficiency, typically $80 \%-$ $85 \%$. Once events are selected, algorithms are applied to reconstruct the four-momenta of the fermions. In $\mathrm{e}^{+} \mathrm{e}^{-} \rightarrow \mathrm{q} \overline{\mathrm{q}} \ell \bar{\nu}_{\ell}$ events, the charged particle tracks and any clusters in the calorimeters (electromagnetic and hadronic) which are not associated with the lepton are forced into two jets using the Durham[9] or LUCLUS[10] algorithms. In $\mathrm{e}^{+} \mathrm{e}^{-} \rightarrow \mathrm{q} \overline{\mathrm{q}} \mathrm{q} \overline{\mathrm{q}}$ events, tracks and clusters are forced into four jets (although DELPHI and OPAL allow for the possibility of a fifth 'gluon' jet). The jet energy resolution is relatively poor, $\sigma_{E} / E>60 \% / \sqrt{E}$, and neutrinos from leptonic W-boson decays are unobserved. Consequently, kinematic fits are employed to improve the event-by-event mass resolution. The kinematic fits impose the constraints of energy

and momentum conservation. In most analyses the additional constraint of equal masses for the two $\mathrm{W}$ bosons is imposed, effectively neglecting $\Gamma_{\mathrm{W}}$, referred to as a five constraint $(5 \mathrm{C})$ fit. The result of the $5 \mathrm{C}$ kinematic fit is a single reconstructed mass for each event which approximates to the average of the invariant masses of the fermion pairs from the two W-boson decays. In general, photons from initial state radiation (ISR) within the detector acceptance are not treated as separate particles in the fit. Although the four LEP collaborations employ 
different procedures to extract $M_{\mathrm{W}}$ from the reconstructed mass distribution, each method essentially corresponds to fitting the peak of the reconstructed mass distribution and calibrating out experimental biases using Monte Carlo event samples.

The single most important aspect of the kinematic fit is the constraint that the sum of the energies of the four fermions is equal to the centre-of-mass energy of the $\mathrm{e}^{+} \mathrm{e}^{-}$collision, $\sqrt{s}$. Due to the poor jet energy resolution, this constraint significantly improves the invariant mass resolution. However, in the presence of ISR, this procedure introduces a bias in the reconstructed mass, since the energies of the four fermions should be constrained to the centreof-mass energy after photon radiation, $\sqrt{s^{\prime}}$, rather than to $\sqrt{s}$. As a result, for events with ISR, the kinematic fit will return a mass value which is too large. On average, the event-by-event mass returned by the kinematic fit approximately corresponds to

$$
\bar{M} \approx \frac{1}{2}\left(M_{12}+M_{34}\right) \times \sqrt{\frac{s}{s^{\prime}}} .
$$

Hence, the reconstructed invariant mass distribution depends strongly on the $\sqrt{s^{\prime}}$ distribution and as a result the peak of reconstructed W-boson mass is approximately $100 \mathrm{MeV}$ higher than $M_{\mathrm{W}}$ (the exact value depending on $\sqrt{s}$ ). The bias in the fitted mass is even greater, typically a few hundred $\mathrm{MeV}$. This bias is subsequently determined using Monte Carlo events and the data measurement corrected. Consequently, deficiencies in the Monte Carlo simulation may result in systematic biases and it is necessary for the Monte Carlo programs to predict accurately the $\sqrt{s^{\prime}}$ distribution.

\section{QED Uncertainties on the LEP $M_{\mathrm{W}}$ Measurement}

The current estimate of $5 \mathrm{MeV}$ for the QED/Electroweak theoretical uncertainty on the LEP $M_{\mathrm{W}}$ measurement is based on the Monte Carlo generated invariant mass distribution of the $\mu^{-} \bar{\nu}_{\mu}$ system in $\mathrm{e}^{+} \mathrm{e}^{-} \rightarrow \mu^{-} \bar{\nu}_{\mu} u \bar{d}(\gamma)$ events[3]. Uncertainties are obtained from the shifts in the invariant mass distribution which result from dropping higher order theoretical terms. The simplest definition of invariant mass is obtained directly from the four-momenta of the generated fermions (termed $B A R E$ mass $^{1}$ ). However, in the real experimental analyses, photons from final state radiation (FSR) are usually recombined with the nearest jet or lepton. This recombination is emulated at the level of the generated particles by recombining photons with the closest fermion if the photon has energy $E_{\gamma}<1 \mathrm{GeV}$ or if the invariant mass of the photon and fermion is less than $5 \mathrm{GeV}(25 \mathrm{GeV})$, termed the CALO5(CALO25) photon recombination scheme. In this way FSR photons are usually recombined with the appropriate fermion. In the $C A L O 5 / C A L O 25$ schemes photons within $5^{\circ}$ of the beam direction are treated as unobserved and are not recombined, reflecting the holes in experimental acceptance along the beam direction.

The main difference between the definitions of $B A R E$ and $C A L O$ masses and the experimentally reconstructed mass arises from the kinematic fit employed in the experimental analyses. As a result of the kinematic fit, $\mathcal{O}(\alpha)$ QED corrections may affect the reconstructed mass in two ways: either by modifying the invariant mass distribution of the fermion pairs from $\mathrm{W}$ decays or by modifying the $\sqrt{s^{\prime}}$ distribution. In the first case, the theoretical estimates of mass biases obtained using either $B A R E$ or $C A L O$ schemes provide a good estimate of the true experimental bias. However, in second case, even if the invariant mass distribution of the

\footnotetext{
${ }^{1}$ The nomenclature of reference [3] is used throughout this paper rather than that of [11].
} 
fermion pairs is unchanged, the experimentally measured mass distribution may be biased as a result of the kinematic fit scaling the measured energies to $\sqrt{s}$ rather than $\sqrt{s^{\prime}}$. Consequently, previous estimates of the impact of theoretical corrections which modify either the rate or energy spectrum of real photons produced in the process $\mathrm{e}^{+} \mathrm{e}^{-} \rightarrow \mathrm{W}^{+} \mathrm{W}^{-} \gamma$ may be too small.

To study possible differences between the previous methods used to estimate $M_{\mathrm{W}}$ systematic uncertainties and the true impact on the experimental measurement the Monte Carlo tandem of KORALW and YFSWW (KandY) is used. A total of four million $\mathrm{e}^{+} \mathrm{e}^{-} \rightarrow \mathrm{W}^{+} \mathrm{W}^{-}$events was generated over 8 separate values of $\sqrt{s}$ reflecting the LEP 2 data sample used in the Wmass determination $(183 \mathrm{GeV}<\sqrt{s}<209 \mathrm{GeV})$. The events were passed through the full OPAL detector simulation[6] and $\mathrm{W}$-mass reconstruction procedure including the 5C kinematic fit[12]. The following studies are based on events passing the OPAL $\mathrm{W}^{+} \mathrm{W}^{-} \rightarrow \mathrm{q} \overline{\mathrm{q}} \ell \bar{\nu}_{\ell}$ and $\mathrm{W}^{+} \mathrm{W}^{-} \rightarrow \mathrm{q} \overline{\mathrm{q}} \mathrm{q} \overline{\mathrm{q}}$ event selections. The KandY program produces correction weights enabling generated events to be reweighted to the corresponding theoretical prediction removing certain terms. Correction weights were produced corresponding to: the replacement of the Screened Coulomb correction[13] with the unscreened correction; degrading the treatment of ISR from $\mathcal{O}\left(\alpha^{3}\right)$ to $\mathcal{O}\left(\alpha^{2}\right)$ YFS exponentiated[14] leading logarithm (LL); and dropping the so-called $\mathcal{O}(\alpha)$ NL electroweak corrections of KandY. It is important to note that the corrections termed ' $\mathcal{O}(\alpha) \mathrm{NL}^{\prime}$ in [3] include bremsstrahlung from the W bosons (referred to as WSR) and the resulting interference with ISR.

The treatment of the Coulomb correction modifies the invariant mass distribution of the fermion pairs from the $\mathrm{W}$ decays. Consequently, the shift in the $B A R E / C A L O$ invariant mass distribution provides a good measure of the true experimental bias. Degrading the treatment of ISR from $\mathcal{O}\left(\alpha^{3}\right)$ to $\mathcal{O}\left(\alpha^{2}\right)$ exponentiated LL has negligible impact on the experimental measurement of $M_{\mathrm{W}}[7]$ (the measurement is sensitive to ISR through a modification of the $\sqrt{s^{\prime}}$ distribution, however the effects of the $\mathcal{O}\left(\alpha^{3}\right)$ terms are small). The effect of the $\mathcal{O}(\alpha) \mathrm{NL}$ electroweak corrections, which include bremsstrahlung from the $\mathrm{W}$ bosons, is more interesting. To study potential changes in distributions it is convenient to define the fractional change in a differential cross section,

$$
\Delta_{N L}=\frac{\left(d \sigma_{K Y}^{N L}-d \sigma_{K Y}\right)}{d \sigma_{K Y}}
$$

where $d \sigma_{K Y}$ is the differential cross section in a given bin from the full KandY generation and $d \sigma_{K Y}^{N L}$ is the corresponding distribution without the $\mathcal{O}(\alpha)$ NL electroweak corrections.

Figure 1 shows the effect of the $\mathcal{O}(\alpha)$ NL corrections on the photon energy and polar angle distribution. These plots are similar to those of Reference [15] showing that the experimental selections do not introduce significant biases for events with additional photons ${ }^{2}$. The inclusion of the $\mathcal{O}(\alpha)$ NL corrections reduces the number of real photons produced in the detector acceptance. The maximum fractional change occurs in the region perpendicular to the beam axis where the $\mathrm{W}^{+} \mathrm{W}^{-} \gamma$ cross section is decreased by $30 \%$. This modification of the photon rate and angular distribution is predominantly due the inclusion of radiation from the W bosons. Specifically, the reduction in the $\mathrm{W}^{+} \mathrm{W}^{-} \gamma$ cross section results from interference between ISR and radiation from the $\mathrm{W}$ bosons ${ }^{3}$. The photon energy spectrum is not greatly distorted by

\footnotetext{
${ }^{2}$ The plots in Reference [15] are for $\mathrm{W}^{+} \mathrm{W}^{-} \rightarrow \mathrm{q} \overline{\mathrm{q}} \ell \bar{\nu}_{\ell} \gamma$ events generated at $\sqrt{s}=200 \mathrm{GeV}$ and do not include the effects of event selection.

${ }^{3}$ This was verified using YFSWW. The reduction in the cross section for photon production shown in Figure 1a was reproduced by switching from $K E Y C O R=2$ to $K E Y C O R=3$ in YFSWW, corresponding to switching from YFS exponentiation for ISR alone to the full YFS form factor including radiation from the W bosons (WSR) and interference between ISR and WSR.
} 
the NL corrections as can be seen from Figure 1.

The potential bias in the measurement of $M_{\mathrm{W}}$ due to the $\mathcal{O}(\alpha)$ NL terms is investigated using four definitions of reconstructed mass were considered:

- $\bar{M}_{B A R E}$ : the average of the two W-boson masses determined from the four-momenta of the four fermions.

- $\bar{M}_{C A L O 5}$ : the average of the two W-boson masses determined from the four-momenta of the four fermions after applying the $C A L O 5$ photon recombination procedure.

- $M_{5 C}$ : the mass returned by the OPAL 5C kinematic fit. Only events for which the fit converges with a probability of greater than $0.1 \%$ are plotted.

- $\bar{M}_{C A L O 5 *}: \bar{M}_{C A L O 5}$ rescaled by $\sqrt{\frac{s}{s^{\prime}}}$ where $\sqrt{s^{\prime}}$ is the invariant mass of the four fermion system after recombination of photons:

$$
\bar{M}_{C A L O 5 *}=\bar{M}_{C A L O 5} \times \sqrt{\frac{s}{s^{\prime}}} .
$$

The CALO5* definition, proposed in this paper, represents an attempt to emulate the effects of the kinematic fit at the level of the four-momenta of the fermions. The distortions of the W-mass peak, plotted for above definitions of invariant mass, are shown in Figure 2. To quantify the effect on the $\mathrm{W}$-mass measurement, the resulting biases in the mean reconstructed W-boson mass (in the region $75 \mathrm{GeV}-90 \mathrm{GeV}$ ) are listed in Table 1. The inclusion of the $\mathcal{O}(\alpha)$ NL corrections has almost no effect on the $B A R E$ mass distribution and the mean value changes by just $1 \mathrm{MeV}$. A small but visible effect is observed for the mass in the CALO5 scheme giving a bias in the mean mass of $3 \mathrm{MeV}$. These results are consistent with those presented in [3]. However, the inclusion of the $\mathcal{O}(\alpha)$ NL corrections produces a significant shift in the experimentally reconstructed mass from the $5 \mathrm{C}$ kinematic fit, with the mean reconstructed mass in the range $75 \mathrm{GeV}-90 \mathrm{GeV}$ shifted by $21 \mathrm{MeV}$. Although the invariant mass distribution of the fermion pairs is almost unaffected the reconstructed mass distribution is significantly biased. The origin of this modification of the $\sqrt{s^{\prime}}$ distribution is the reduction in the production cross section for $\mathrm{e}^{+} \mathrm{e}^{-} \rightarrow \mathrm{W}^{+} \mathrm{W}^{-} \gamma$, which results from the interference of ISR and radiation from the $\mathrm{W}$ bosons. It should be noted that by scaling the mean mass in the CALO5 scheme by $\sqrt{s / s^{\prime}}$ (denoted $C A L O 5 *$ ) the effect of the kinematic fit can be approximated at the level of the generated fermions.

The effect of the kinematic fit is to make the experimental analyses more sensitive to theoretical corrections which modify the rate/spectrum of photons produced in the process $\mathrm{e}^{+} \mathrm{e}^{-} \rightarrow \mathrm{W}^{+} \mathrm{W}^{-} \gamma$. The bias of $21 \mathrm{MeV}$ associated with the $\mathcal{O}(\alpha) \mathrm{NL}$ corrections in KandY is almost an order of magnitude larger than previously quoted. Since the origin of the $21 \mathrm{MeV}$ bias is interference between ISR and radiation from the $\mathrm{W}$ bosons, one might expect this to be well accounted for in YFSWW, but this requires further evaluation. It should be noted that the $21 \mathrm{MeV}$ bias would be reduced significantly if observed photons were treated as additional particles in the kinematic fits. In this case the energies of the fermions from $\mathrm{W}$ decay would be scaled correctly to $\sqrt{s^{\prime}}$.

For the OPAL W-mass extraction procedure the effect of the $\mathcal{O}(\alpha)$ NL corrections on the full $M_{\mathrm{W}}$ reconstruction procedure is somewhat smaller than the shift in the average event-by-event reconstructed mass quoted above. Using a modified version of the OPAL $M_{\mathrm{W}}$ convolution fit[7] the bias associated with dropping the $\mathcal{O}(\alpha)$ NL corrections is $14 \mathrm{MeV}$. 


\begin{tabular}{|l|r|}
\hline Scheme & $\Delta M^{N L}$ \\
\hline BARE & $+1 \mathrm{MeV}$ \\
CALO5 & $+3 \mathrm{MeV}$ \\
CALO5* & $+22 \mathrm{MeV}$ \\
5C FIT & $+21 \mathrm{MeV}$ \\
\hline
\end{tabular}

Table 1: The change in the mean $\mathrm{W}$-boson mass in the range $75 \mathrm{GeV}-90 \mathrm{GeV}$ when dropping the electroweak $\mathcal{O}(\alpha)$ NL corrections from KandY. Shifts are shown for three different theoretical definitions of the event-by-event reconstructed mass: BARE, CALO5 and the new definition proposed in this paper, CALO5*. 5C FIT refers to the shift in the average of reconstructed mass distribution for the full OPAL event reconstruction using a $5 \mathrm{C}$ kinematic fit.

\section{Limits from the Measurement of the $\mathrm{e}^{+} \mathrm{e}^{-} \rightarrow \mathrm{W}^{+} \mathrm{W}^{-} \gamma$ Cross Section}

Measurements of the process $\mathrm{e}^{+} \mathrm{e}^{-} \rightarrow \mathrm{W}^{+} \mathrm{W}^{-} \gamma$ have been performed at LEP $[16,17,18]$. These measurements, which are restricted to photons within the experimental acceptance (typically $\left.\left|\cos \theta_{\gamma}\right|<0.95\right)$, provide a direct probe of $\mathcal{O}(\alpha)$ QED corrections which affect real photon production away from the region collinear with the $\mathrm{e}^{+} \mathrm{e}^{-}$beams. Of particular interest here are the $\mathcal{O}(\alpha)$ NL electroweak corrections of KandY which decrease the calculated $\mathrm{e}^{+} \mathrm{e}^{-} \rightarrow \mathrm{W}^{+} \mathrm{W}^{-} \gamma$ cross section. The largest change in the differential $\mathrm{W}^{+} \mathrm{W}^{-} \gamma$ cross section occurs close to the beam direction (as would be expected from ISR-WSR interference). However, when integrated over the photon polar angle, $88 \%$ of the change in cross section occurs within the nominal experimental acceptance of $\left|\cos \theta_{\gamma}\right|<0.95$. Hence, by measuring the $\mathrm{e}^{+} \mathrm{e}^{-} \rightarrow \mathrm{W}^{+} \mathrm{W}^{-} \gamma$ cross section for events where the photon is observed, it is possible to probe experimentally the $\mathcal{O}(\alpha)$ NL corrections of KandY.

When the four LEP experiments publish final results including all LEP 2 data, the combined experimental precision on the $\mathrm{W}^{+} \mathrm{W}^{-} \gamma$ cross section should be approximately $7 \%$. The inclusion of the $\mathcal{O}(\alpha)$ NL electroweak corrections of KandY produces a $20 \%$ decrease in the $\mathrm{e}^{+} \mathrm{e}^{-} \rightarrow \mathrm{W}^{+} \mathrm{W}^{-} \gamma$ cross section for $\left|\cos \theta_{\gamma}\right|<0.95$ and $E_{\gamma}>5 \mathrm{GeV}$ (cuts chosen to match the experimental acceptances of the LEP detectors). Thus, using rate alone, the measurements are of sufficient statistical precision to provide a useful test of the KandY implementation of WSR and in particular of WSR-ISR interference. Agreement with the predictions of KandY would provide the first indirect observation (albeit only at the $3 \sigma$ level) of real photon radiation from the $\mathrm{W}$ bosons through interference with ISR diagrams. The statistical precision of this test would be improved slightly by including angular information in addition to the photon rate.

In the context of the $M_{\mathrm{W}}$ measurement at LEP the inclusion of the $\mathcal{O}(\alpha)$ NL corrections in KandY produces a $21 \mathrm{MeV}$ shift in the mean reconstructed mass which results from the modification of the $\sqrt{s^{\prime}}$ distribution. This is a direct consequence of the decrease in the $\mathrm{e}^{+} \mathrm{e}^{-} \rightarrow$ $\mathrm{W}^{+} \mathrm{W}^{-} \gamma$ cross section. Therefore, measurements of the $\mathrm{e}^{+} \mathrm{e}^{-} \rightarrow \mathrm{W}^{+} \mathrm{W}^{-} \gamma$ cross section at LEP provide constraints on the $\mathcal{O}(\alpha)$ QED uncertainties related to real photon production away from the region collinear with the beams. A $20 \%$ reduction in the $\mathrm{W}^{+} \mathrm{W}^{-} \gamma$ cross section corresponds to a $21 \mathrm{MeV}$ shift in the reconstructed value of $M_{\mathrm{W}}$. Assuming the bias scales linearly, a measurement of the $\mathrm{W}^{+} \mathrm{W}^{-} \gamma$ cross section with a statistical precision of $7 \%$ would correspond 
to approximately $7 \mathrm{MeV}$ on the associated systematic uncertainty on $M_{\mathrm{W}}$. It should be noted that this limit would apply to all theoretical corrections to the process $\mathrm{e}^{+} \mathrm{e}^{-} \rightarrow \mathrm{W}^{+} \mathrm{W}^{-}$which modify the rate of real photon production away from the collinear region; it is not specific to the $\mathcal{O}(\alpha)$ NL corrections of KandY. Given that the expected statistical uncertainty on the LEP $M_{\mathrm{W}}$ measurement is approximately $35 \mathrm{MeV}$, a LEP combined measurement of the $\mathrm{W}^{+} \mathrm{W}^{-} \gamma$ cross section would provide a useful experimental constraint on the $\mathcal{O}(\alpha)$ QED systematic uncertainty.

\section{Conclusions}

Previous studies of QED systematic uncertainties on the LEP measurement of $M_{\mathrm{W}}$ have used idealized event selections and fitting procedures. In this paper the KandY Monte Carlo program was used to study the effect of the experimental methodology on the estimated QED systematic uncertainty. It is found that the kinematic fitting used in the experimental determination of $M_{\mathrm{W}}$ enhances the sensitivity to all QED corrections involving real photon production and it is concluded that previous estimates of the QED theoretical uncertainty on the LEP 2 $M_{\mathrm{W}}$ measurement may be too small. A simple procedure for approximating the effect of the kinematic fit at the level of the generated four fermions is proposed. This procedure would allow previous theoretical studies to be extended to include a good approximation of the real experimental mass extraction method. Finally, it is shown that useful experimental limits on $\mathcal{O}(\alpha)$ theoretical uncertainties related to real photon production away from region collinear with the $\mathrm{e}^{+} \mathrm{e}^{-}$beams may be inferred from LEP measurements of the $\mathrm{e}^{+} \mathrm{e}^{-} \rightarrow \mathrm{W}^{+} \mathrm{W}^{-} \gamma$ cross section.

\section{Acknowledgments}

I would like acknowledge the authors of the KandY program, S. Jadach, W. Płaczek, M. Skrzypek, B.F.L. Ward and Z. Wạs, without whom this study would not have been possible. In particular I thank M. Skrzypek and W. Płaczek for their many useful comments and suggestions. I also would like to thank my colleagues on the OPAL collaboration for allowing me to present results using the OPAL detector simulation and W mass analysis tools. In particular M. Verzocchi for his work in generating the KandY Monte Carlo samples and R. Hawkings for providing results from the full OPAL $M_{\mathrm{W}}$ fit. Finally I thank D.R. Ward and C.P. Ward for reading through draft versions of this paper.

\section{References}

[1] Program YFSWW3, S. Jadach et al., Phys. Lett. B417 (1998) 326.

[2] Program RacoonWW, A. Denner, S. Dittmaier, M. Roth and D. Wackeroth, Nucl. Phys. B560 (1999) 33;

A. Denner, S. Dittmaier, M. Roth and D. Wackeroth, Nucl. Phys. B587 (2000) 67.

[3] S. Jadach et al., Phys. Lett. B523 (2001) 117.

[4] Program KORALW V1.51 and YFSWW3, S. Jadach et al., Comp. Phys. Comm. 140 (2001) 475 . 
[5] Program KORALW V1.42, M. Skrzypek et al., Comp. Phys. Comm. 94 (1996) 216;

M. Skrzypek et al., Phys. Lett. B372 (1996) 289;

M. Skrzypek et al., Comp. Phys. Comm. 119 (1999) 1.

[6] J. Allison et al., Nucl. Instr. and Meth. A317 (1992) 47.

[7] OPAL Collaboration, G. Abbiendi et al., Phys. Lett. B507 (2001) 29.

[8] L3 Collaboration M. Acciarri et al., Phys. Lett. B454 (1999) 386;

ALEPH Collaboration, R. Barate et al., Eur. Phys. J. C17 (2000) 241;

DELPHI Collaboration, P. Abreu et al.Phys. Lett. B515 (2001) 238.

[9] N. Brown and W. J. Stirling, Phys. Lett. B252 (1990) 657;

S. Bethke, Z. Kunszt, D. Soper and W. J. Stirling, Nucl. Phys. B370 (1992) 310;

S. Catani et al., Phys. Lett. B269 (1991) 432;

N. Brown and W.J. Stirling, Z. Phys. C53 (1992) 629.

[10] T. Sjöstrand, Pythia 5.7 and Jetset 7.4, Physics and Manual, CERN-TH-7112-93-REV (1995).

[11] M. W. Grunewald and G. Passarino et al., "Four-Fermion Production in Electron-Positron Colliders", CERN-2000-09-A (hep-ph/0005309).

[12] OPAL Collaboration, G. Abbiendi et al., Eur. Phys. J. C1 (1998) 395.

[13] A. P. Chapovsky and V. A. Khoze, Eur. Phys. J. C9 (1999) 449.

[14] D. R. Yennie, S. C. Frautschi and H. Suura, Ann. Phys. 13 (1961) 379.

[15] Figure 9 of reference [4].

[16] OPAL Collaboration, G. Abbiendi et al., Phys. Lett. B471 (1999) 293.

[17] L3 Collaboration, P. Achard et al., Phys. Lett. B527 (2002) 29.

[18] Delphi Collaboration, Measurement of the $\mathrm{W}^{+} \mathrm{W}^{-} \gamma$ cross-section and limits on anomalous quartic gauge couplings from DELPHI, Contributed paper to ICHEP 2002, Amsterdam, July 2002. 

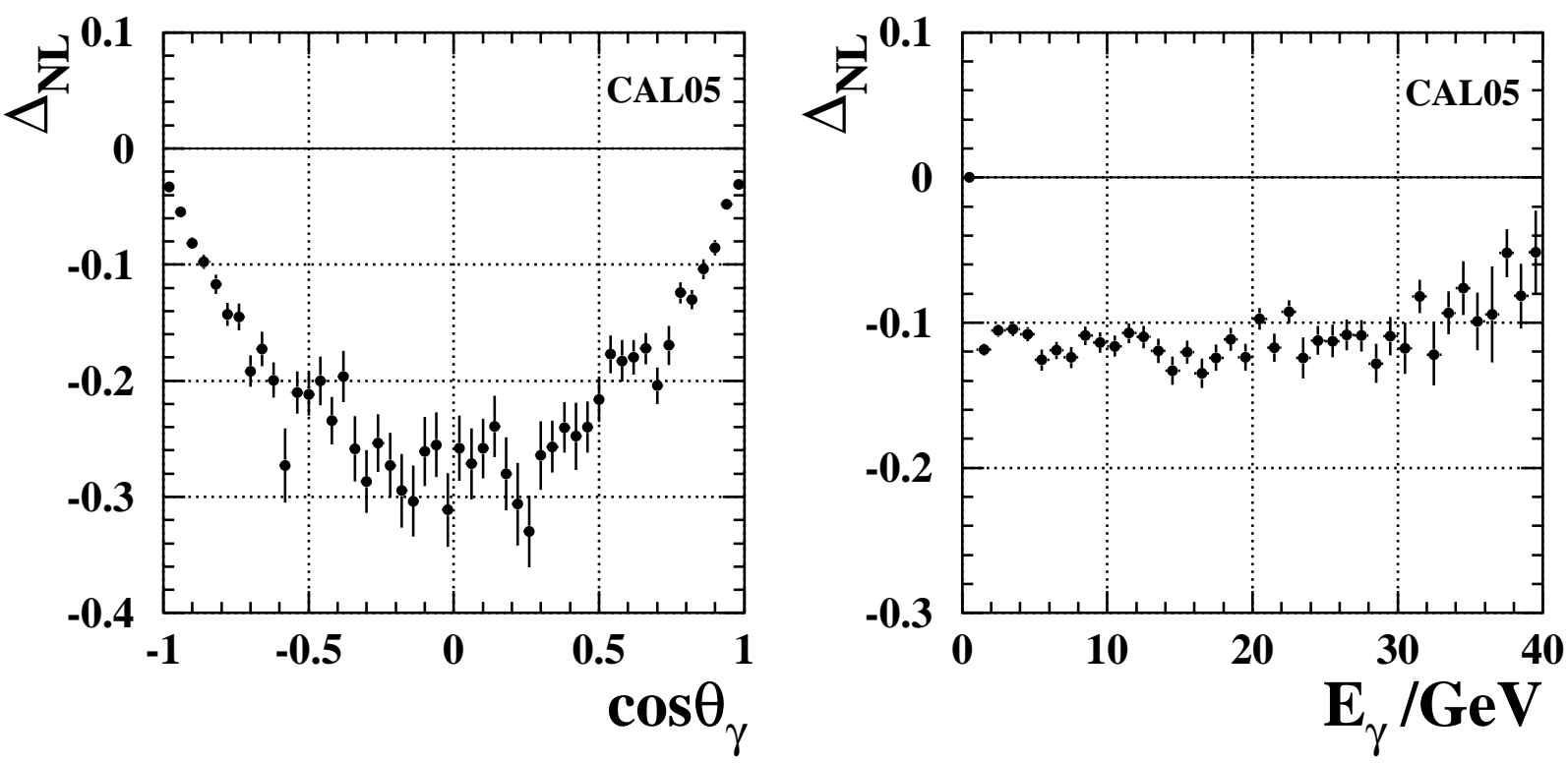

Figure 1: The effect of the $\mathcal{O}(\alpha)$ NL corrections in KandY on the distributions of the energy and angle with respect to the $e^{-}$beam of the highest energy photon in the event. Only photons which have not been recombined using CALO5 scheme are included. 

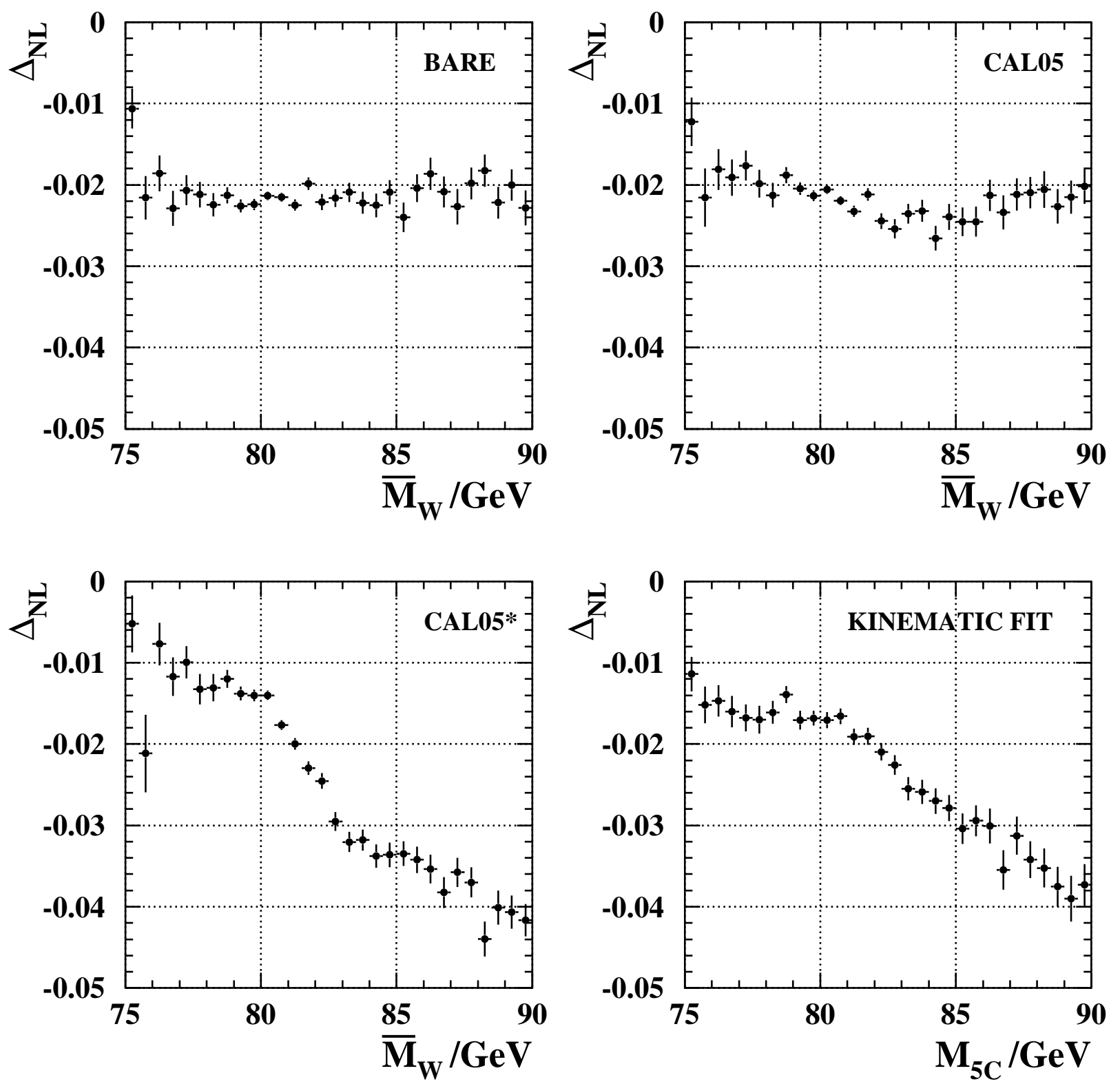

Figure 2: Fractional change in the differential cross-section as a function of the reconstructed mass using the BARE, CALO5, and CALO5* schemes compared to the experimental bias using the OPAL $5 \mathrm{C}$ kinematic fit. 